\title{
Physical properties and evolutionary states of binaries and variable stars observed by LAMOST
}

\author{
Shengbang Qian*, Liying Zhu, Ergang Zhao, Xiangdong Shi, Zhihua, Wang \\ Yunnan Observatories, Chinese Academy of Sciences, 396 Yangfangwang, Guandu District, \\ Kunming, 650216, P. R. China \\ Center for Astronomical Mega-Science, Chinese Academy of Sciences, 20A Datun Road, \\ Chaoyang District, Beijing, 100012, P. R. China \\ Key Laboratory for the Structure and Evolution of Celestial Objects, Chinese Academy of \\ Sciences, 396 Yangfangwang, Guandu District, Kunming, 650216, P. R. China \\ University of the Chinese Academy of Sciences, Yuquan Road 19\#, Sijingshang Block, 100049 \\ Beijing, P. R. China \\ E-mail: Gsbdynao.ac.cn
}

\begin{abstract}
About eighteen thousand known variables were observed by LAMOST during the first stage of the spectroscopic survey from October 24, 2011 to July 16, 2017. Stellar atmospheric parameters for about eleven thousands of them were determined including the temperature $(\mathrm{T})$, the gravitational acceleration $(\log \mathrm{g})$, the metallicity $[\mathrm{Fe} / \mathrm{H}]$ and the radial velocity $V_{\mathrm{r}}$. Those parameters are introduced in the paper. The locations of those variables including $\delta$ Sct and $\gamma$ Dor stars on the Log g-T diagram are presented where EA- and EW-type eclipsing binaries are also shown for comparison. Meanwhile, new photometric results on two special sample stars ROTSE1 J144111.82+382750.0 and CSS_J034112.5+035336 were obtained by follow-up observations. The former was originally found as a $\delta$ Sct with a period of 0.191291 days. However, the LAMOST parameters reveal that it is far beyond the red edge of pulsational instability trip of $\delta$ Sct stars. By using those new photometric data, we detect that is an EW-type eclipsing binary with an orbital period of 0.382583. As for CSS_J034112.5+035336, it was listed as an EW-type binary in VSX with a period of 0.285554 days. However, the LAMOST data showed that it is a special target because its temperature is very higher when compared with the short period. Our new photometric observations reveal it is a pulsating star rather than an EW-type binary. These results indicate that they the LAMOST data are very useful to understand their physical properties and evolutionary states.
\end{abstract}

Accretion Processes in Cosmic Sources - II - APCS2018

3-8 September 2018

Saint Petersburg, Russian Federation

\footnotetext{
* Speaker.
} 


\section{Introduction}

Variable stars including eclipsing binaries (EBs) are very important sources in astrophysics. EBs could be used to determine the most reliable stellar parameters (e.g., [ [U, []] and references therein). Their strong interactions between both components could produce many astrophysical processes such as mass transfer and accretion, common envelope and different stellar outbursts. On the other hand, pulsating stars are a good source to study stellar internal structure and stellar evolution. Thanks to several photometric surveys, such as Catalina Sky Survey (CSS, [B], 团]), the asteroid survey LINEAR ([[]]), All Sky Automated Survey (ASAS, [6, 四]) and Northern sky variability survey (NSVS, [ $[8]$ ), a large number of variable stars together with many EBs were discovered. They were listed in the international variable star index (VSX) that is to bring all of the new information together in a single data repository ([Q]]). However, the spectroscopic data of those variables are extremely lacking because no efficient spectroscopic surveys contributed on stars.

Large Sky Area Multiobject Fiber Spectroscopic Telescope (hereafter LAMOST) is a special telescope that has an aperture about 4 meters with a field of view about 5 degree ([ए], प]). It is mainly used for low-resolution spectroscopic survey with a spectral wavelength range from 3700 to $9000 \AA$ A. In one exposure, the spectra of about 4000 stars could be obtained by LAMOST. In the time interval between October 24, 2011 and July 16, 2017, more than three millions stars were observed by LAMOST. During the first stage of spectroscopic survey from October 24, 2011 to July 16, 2017, huge amounts of spectroscopic data were obtained by LAMOST (e.g., [12], [3]) including pulsating stars ([4], [5]) and binary systems ([ए], [0] ]). In Section 2, those stellar atmospheric parameters for variables and binary stars are introduced and their Log g-T diagram is presented. The detection of a new EW-type eclipsing binary by follow-up photometric observations is presented in Section 3. The finding of a new pulsating star is introduced in Section 4. Finally, discussions of the results and conclusions are given in Section 6.

\section{Variable stars or eclipsing binaries observed by LAMOST}

During the first stage of LAMOST spectroscopic survey, about 18000 known variables and binaries were observed by LAMOST from October 24, 2011 to July 16, 2017. Among the 18000 stars, the stellar atmospheric parameters for about 11000 variables or binaries were determined when their spectra have higher signal to noise, while only spectral types were obtained for the rest ones. Those stellar parameters include the effective temperature $T$, the gravitational acceleration $\log (\mathrm{g})$, the metallicity $[\mathrm{Fe} / \mathrm{H}]$ and the radial velocity $V_{r}$. They were automatically determined by the LAMOST stellar parameter pipeline (e.g., [ㅈ]]) that is based on the ULySS (Universite de Lyon spectroscopic analysis software) (e.g., [18]]). The standard deviations are $110 \mathrm{~K}$ for the temperature, 0.19 dex for the gravitational acceleration and 0.11 dex for the metallicity when $T_{\text {eff }}<8000 \mathrm{~K}$. As for the radial velocity $V_{r}$, when $T_{\text {eff }}<10000 \mathrm{~K}$, the standard deviations are $4.91 \mathrm{Km} / \mathrm{s}$ (e.g., [प्व]). The comparison between the LAMOST temperatures with those listed in the Kepler input catalogue is plotted in Fig. 1 were about 12221 stars in the LAMOST-Kepler field are shown. As displayed in the figure, there is a good agreement for the temperature. The dashed blue lines refer to a difference of $200 \mathrm{~K}$ for $\mathrm{T}$ and most of the targets are within the differences. 


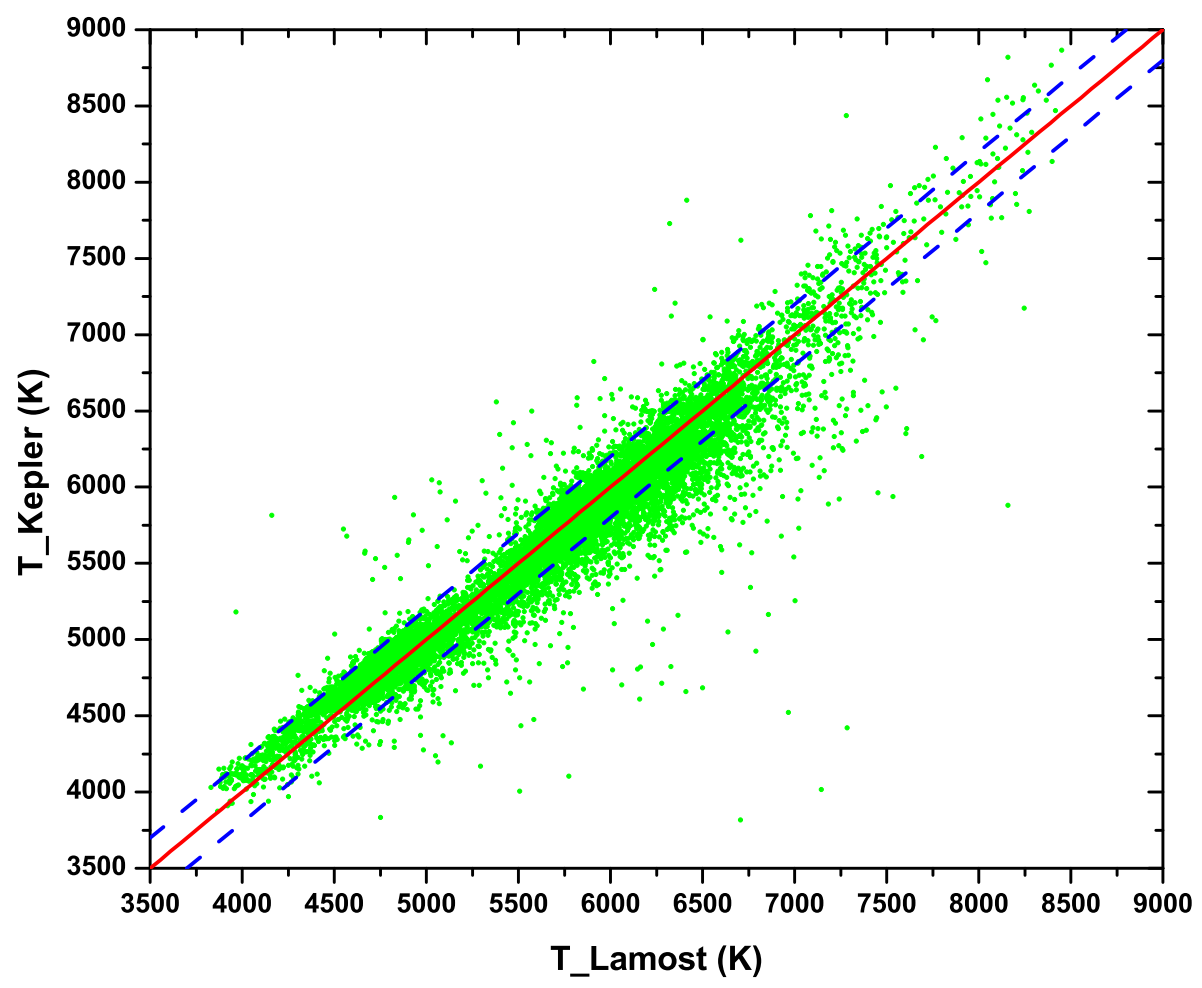

Figure 1: Comparison between the LAMOST temperatures with those listed in the Kepler input catalogue for 12221 tstars in the LAMOST-Kepler field. The red solid line is the one-to-one relationship. The green dashed lines refer to a difference of $200 \mathrm{~K}$.

Based on the LAMOST data of $\delta$ Scuti stars, Qian et al.[14] found a special group of unusual and cool variable stars (UCVs) with effective temperatures below $6700 \mathrm{~K}$ and periods in the range from 0.09 to 0.22 days. The UCVs are far beyond the red edge of pulsational instability trip on the H-R diagram and the $\log g-T$ diagram and they are distinguished from the normal $\delta$ Scuti stars (NDSTs). After those UCVs are excluded, Qian et al. [14] discovered that the effective temperature, the gravitational acceleration and the metallicity all are correlated with the pulsating period for NDSTs. A recent investigation by Qian et al. [प5] reveals that the physical properties of $\gamma$ Dor (GDOR) stars are the same as those of long-period $\delta$ Scuti $(P>0.3$ days) stars. There are no such these relations for long-period pulsating stars with periods longer than 0.3 days including $\gamma$ Dor stars and long-period $\delta$ Scuti variables. The locations of those variables on the Log g-T diagram are presented in Fig 2. For comparison, EA- and EW-type eclipsing binaries observed by LAMOST are also shown in the figure where about 7900 EAs or EWs are displayed. Those LAMOST data of EWs and EAs were used by Qian et al. [ए6, [17] to study their physical properties and their evolutionary relationships. It was shown that EAs are the progenitors of EWs and the modern EW populations could be divided into three groups that may be formed through different mechanisms. As shown in the figure, most of those variables and binaries are main-sequence stars or subgiants. These results indicate that the LAMOST data are very useful to understand their physical properties and evolutionary states. 


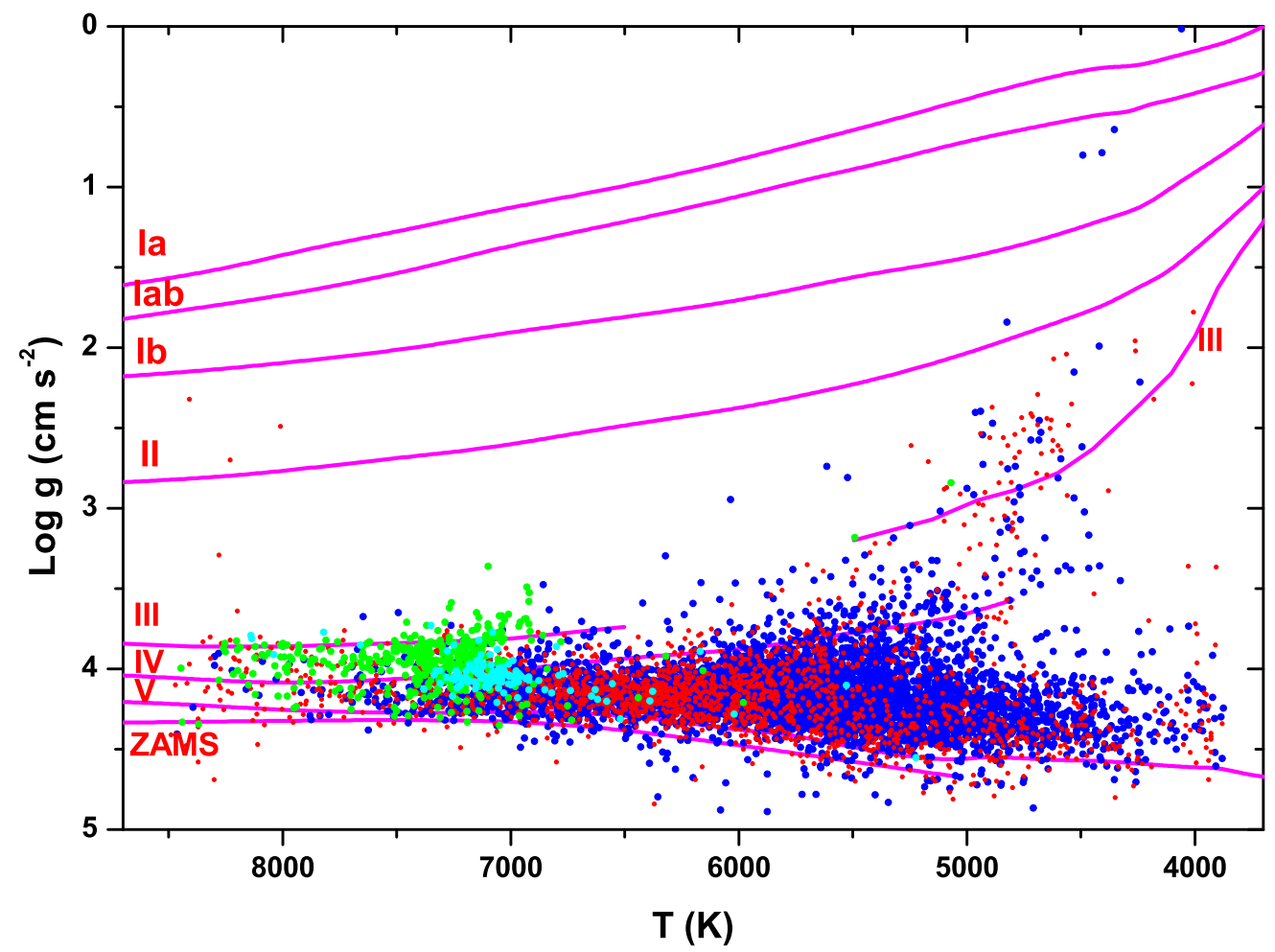

Figure 2: Comparisons between different types of pulsating stars and eclipsing binaries in the Log (g)-T diagram. The magenta lines stand for the luminosity classes range between Ia and $\mathrm{V}$ that are from Straizys \& Kuriliene [ㅈ] , while the zero-age main-sequence line is from Cox [D]]. Green and cyan dots refer to NDSCT and GDOR variables, while blue and red dots to EW- and EA-type eclipsing binary stars.

\section{Detection of a new EW-type eclipsing binary}

ROTSE1 J144111.82+382750.0 (hereafter J144111) was detected as a $\delta$ Sct star with a pulsating period of 0.191291 days (e.g., [ए2] . It was also listed as periodic variable star in the Catalina surveys ([䧃]). J144111 was later observed by LAMOST on January 28, 2014 during the first stage of the spectroscopic survey. Stellar atmospheric parameters were derived as $T=5800 \pm 170 \mathrm{~K}$, $\log g=4.30 \pm 0.24,[\mathrm{Fe} / \mathrm{H}]=-0.48 \pm 0.16$ and $V_{\mathrm{r}}=-58 \pm 13 \mathrm{Km} / \mathrm{s}$. Qian et al. [प]] detected that it belongs one of a group of 131 unusual and cool variable stars (UCVs). Its position on the $\log g-T$ diagram is shown in Fig. 3 as the green triangle. As shown in the figure, it is far beyond the red edges of pulsational instability trip given by both Rodríguez \& Breger [R2] and by Xiong et al. [24]. This indicates that it may be a special variable or a misclassified star.

To understand its physical properties and evolutionary state, it was observed in May 2017 and June 2018 by using the $85-\mathrm{cm}$ telescope at Xinglong Station of National Astronomical Observatories. This telescope is equipped with a CCD camera and the standard Johnson-Cousin-Bessel $B V R_{c} I_{c}$ filters ([[25]). The obtained light curves in $B V R_{c}$ bands are show in Fig. 4 where the phases of those data are calculated with the following linear ephemeris,

$$
\operatorname{Min} . I(H J D)=2457899.2026+0^{d} .382582 \times E .
$$




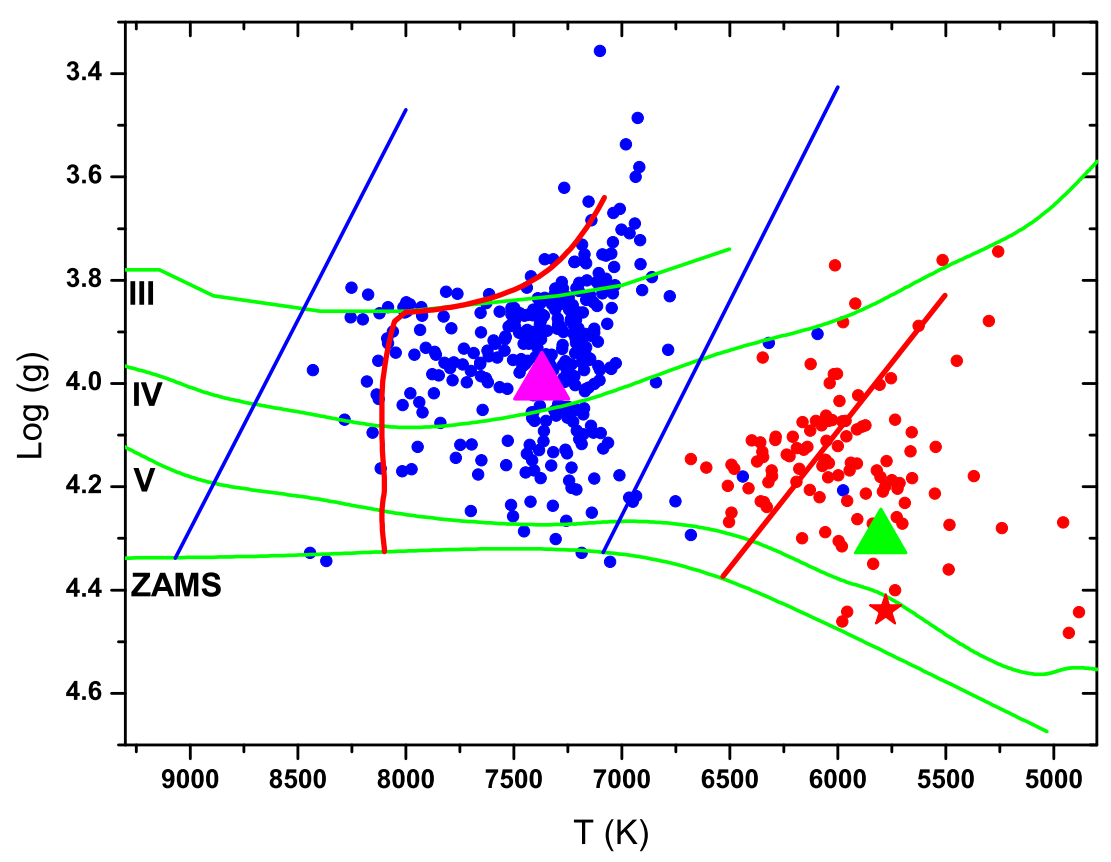

Figure 3: The position of $\mathrm{J} 144111$ on the $\log g-T$ diagram (the green triangle). Blue and red dots refer of NDSTs and UCVs, respectively. The red star is the position of the Sun. The blue lines represent the blue and red edges of $\delta$ Scuti instability trip from Rodríguez \& Breger [D23], while the red lines are the blue and red edges determined by Xiong et al. [24]. The luminosity classes (green lines) and the zero-age main sequence are the same as those in Fig. 2. Also shown as the magenta triangle is the new detected pulsating star J034112.

As shown in the figure, the light curves are typical EW type where the light variation is continuously and the depths of the two minima are nearly the same. The two maxima are asymmetric and are showing a typical O'Connell effect $([2]])$ where the maximum follows the primary minimum is slightly lower than the other one. These results suggest that J14411 is a new EW-type contact binary star.

\section{Detection of a new pulsating star}

CSS_J034112.5+035336 (hereafter J034112) was included in the century survey galactic halo project III ([[2]]). It was classified as an EW-type eclipsing binary in the Catalina surveys ([四]). Its orbital period was derived as 0.285554 days that is close to the peak of the period distribution for EWs. The magnitude in $\mathrm{V}$ is about 14.1 with an amplitude of 0.17 . During the first stage of LAMOST spectroscopic survey, J034112 was observed on December 11, 2014. Stellar atmospheric parameters determined with LAMOST are: $T=7370 \pm 20 \mathrm{~K}, \log g=4.00 \pm 0.03$, $[\mathrm{Fe} / \mathrm{H}]=-0.20 \pm 0.02$ and $V_{\mathrm{r}}=+18 \pm 3 \mathrm{Km} / \mathrm{s}$. The spectral type was classified as $\mathrm{A} 7$ that is too 


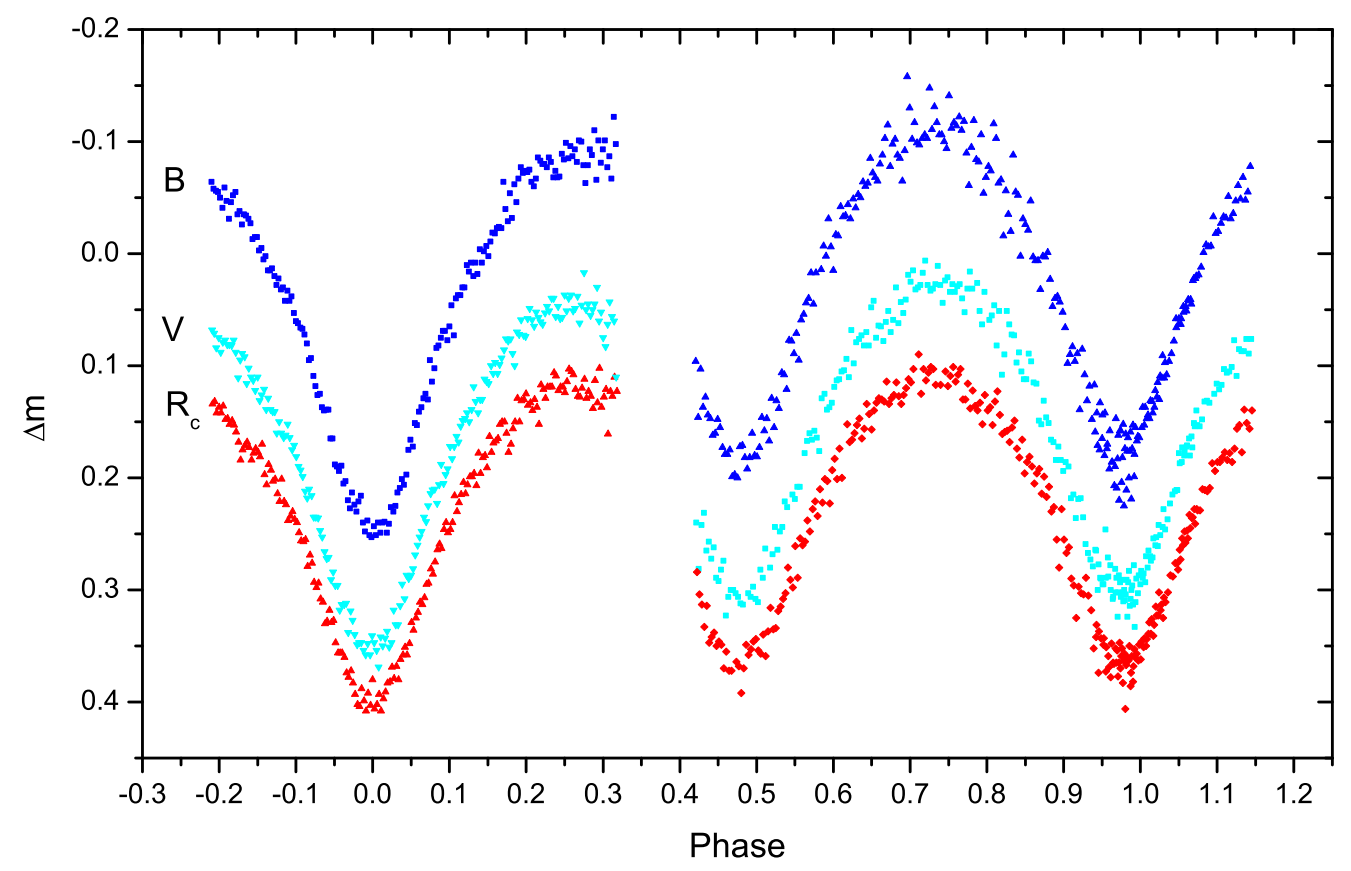

Figure 4: The $B V R_{c}$ light curves of $\mathrm{J} 144111$ obtained with the $85-\mathrm{cm}$ telescope. It is shown that it is a typical EW-type eclipsing binary.

earlier when compared with it short period.

By using the LAMOST data, the relations between the orbital period and the temperature for EW- and EA-type binaries were given by Qian et al. [1저, []]. The position of J034112 on the P-T diagram is plotted in Fig. 5 as the magenta star. As we can see in the figure, it shows a large deviation from the general trend formed by other EWs. This indicates that this target may contain a massive third body or it was misclassified. New photometric data of J034112 were obtained in two successive nights in November 2018 by using the 2.4-m telescope at the Lijiang observational station of Yunnan Observatories (YNOs). During the observations, YFOSC (Yunnan Faint Object Spectrograph and Camera, $2 K \times 4 K$ ) and $\mathrm{BVR}_{c} \mathrm{I}_{c}$ filters were used that are close to the broad-band Johnson-Cousins system. PHOT (measure magnitudes for a list of stars) of the aperture photometry package of IRAF was applied to reduce the observed CCD images.

The $\mathrm{BVR}_{c} \mathrm{I}_{c}$ light curves of J034112 observed on December 18 and 19 are displayed in Figs. 6 and 7 along with the HJD. As shown in the two figures, they are similar to those of pulsating stars where the light maxima are asymmetric. The B-band light curve has the largest amplitude and the light amplitudes are decreasing as the wavelength increases. When compared the light curves obtained on December 18 with those observed on December 19, it is shown the maxima are increasing that indicates the light curve is variable. These properties are usually encountered for pulsating stars. 


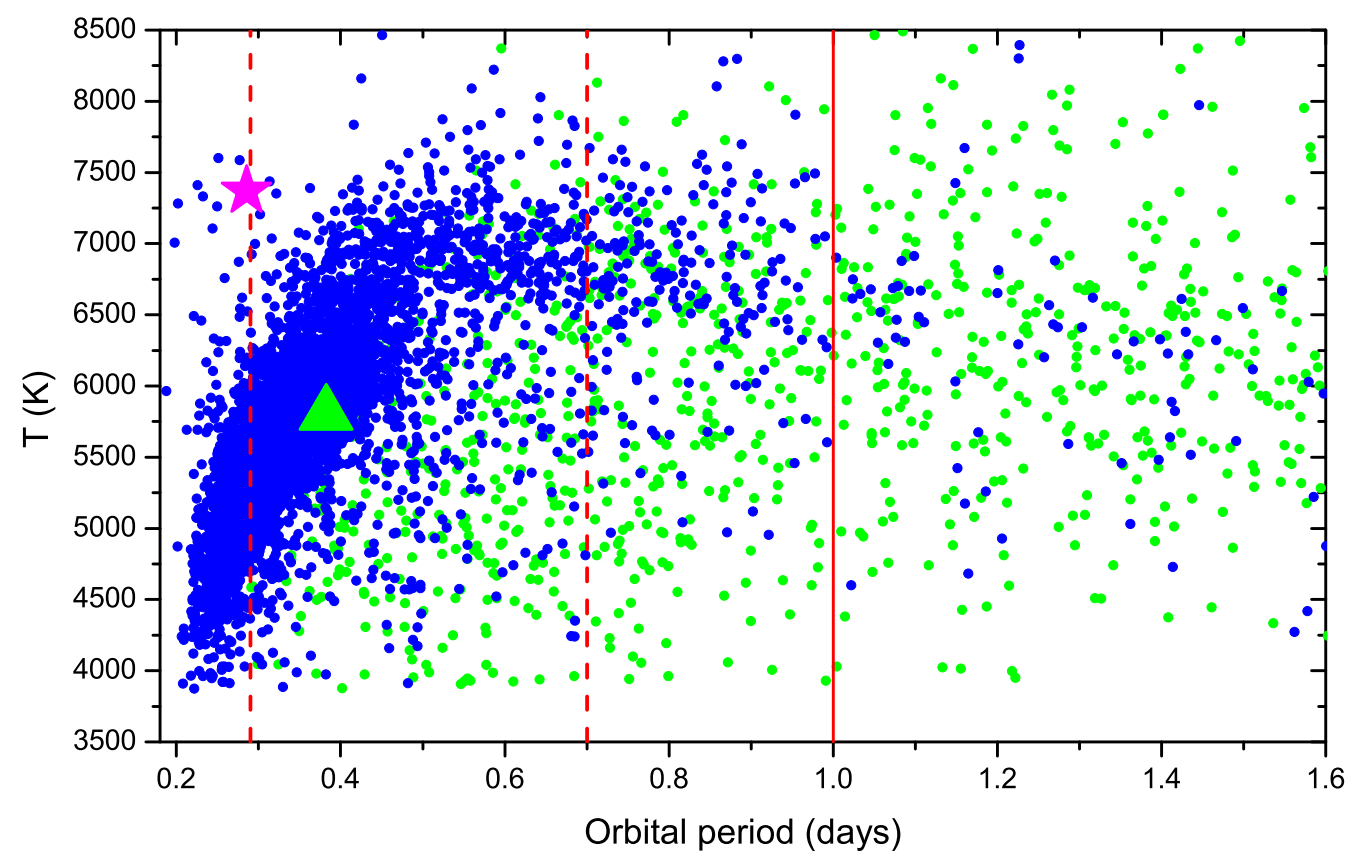

Figure 5: The position of J034112 (the magenta star) on the P-T diagram. Blue dots refer to EWs, while green ones to EAs. Only binary systems with orbital period shorter than 1.6 days are shown. The red solid line indicates that most EWs are short-period systems with period below one day. The red dashed line refer to the peaks of period distributions for EAs and EWs given by Qian et al. [ㅁ] ]. Also shown as the large green triangle is the position of the new detected EW-type binary J144111.

\section{Discussions and conclusions}

In the past several years from October 24, 2011 to July 16, 2017, about 18000 known variable stars and binaries were observed by LAMOST during the first stage of the spectroscopic survey. Stellar atmospheric parameters including the temperature $(\mathrm{T})$, the gravitational acceleration (log $\mathrm{g}$ ), the metallicity $[\mathrm{Fe} / \mathrm{H}]$ and the radial velocity $V_{\mathrm{r}}$ were determined for about 11000 of them, while only spectral types were obtained for the rest ones. The locations of those $\delta$ Sct and $\gamma$ Dor stars on the Log g-T diagram are shown in Fig. 2 where EA- and EW-type eclipsing binaries are also presented for comparison. Most of those variables and binaries are main-sequence stars or subgiants with temperatures below $8500 \mathrm{~K}$ and their parameters could be determined in high precision. Although large number of variable stars and EBs have been discovered (e.g., [Q] ) by several photometric surveys in the world, their spectroscopic information is extremely lacking. Those LAMOST data provide valuable information on the investigating their physical properties and evolutionary states.

New photometric results on the two special sample stars J144111 and J034112 were obtained by follow-up observations. J144111 was originally found as a $\delta$ Sct with a period of 0.191291 days. 


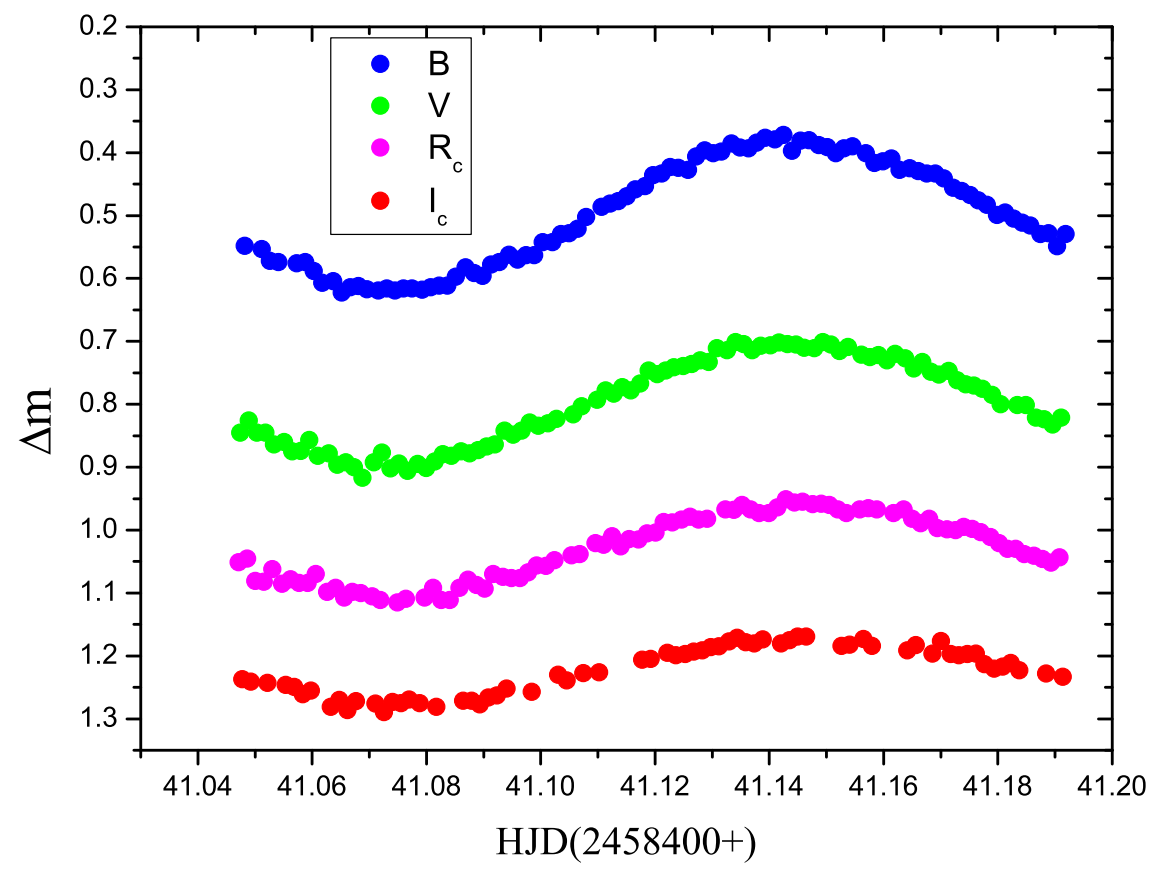

Figure 6: $B V R_{c} I_{c}$ light curves of J034112 obtained on November 18, 2018 by using 2.4-m telescope.

However, Qian et al. [14] found that it belongs one of UCVs. On the $\log g-T$ diagram, it is far beyond the red edges of pulsational instability trip for $\delta$ Sct stars. Our $B V R_{c}$ light curves reveal that is an EW-type eclipsing binary with an orbital period of 0.382583 . The position of J144111 on the P-T diagram is plotted in Fig. 5 as the green triangle. As shown in the figure, it is in agreement with the general trend formed by other EWs. This confirms that this target really EW-type contact binary and needs further observations and investigations.

As for the other target J034112, it was reported as an EW-type binary with a period of 0.285554 days. However, the temperature determined by LAMOST is very higher when compared with its short period and the spectral type is too earlier. Our new $\mathrm{BVR}_{c} \mathrm{I}_{c}$ light curves are similar to those of pulsating stars. It is found that the light amplitudes are decreasing as the wavelength increases and the maxima of those light curves are increasing that indicates the light curve is variable. The position of J034112 on the $\log g-T$ diagram is plotted as the magenta triangle in Fig. 3 where many $\delta$ Sct stars are located and it is slightly evolved from main sequence. All of the results suggest that it is a new detected pulsating star rather than an EW-type binary.

\section{Acknowledgments}

Guoshoujing Telescope (the Large Sky Area Multi-Object Fiber Spectroscopic Telescope LAMOST) is a National Major Scientific Project built by the Chinese Academy of Sciences. Funding 


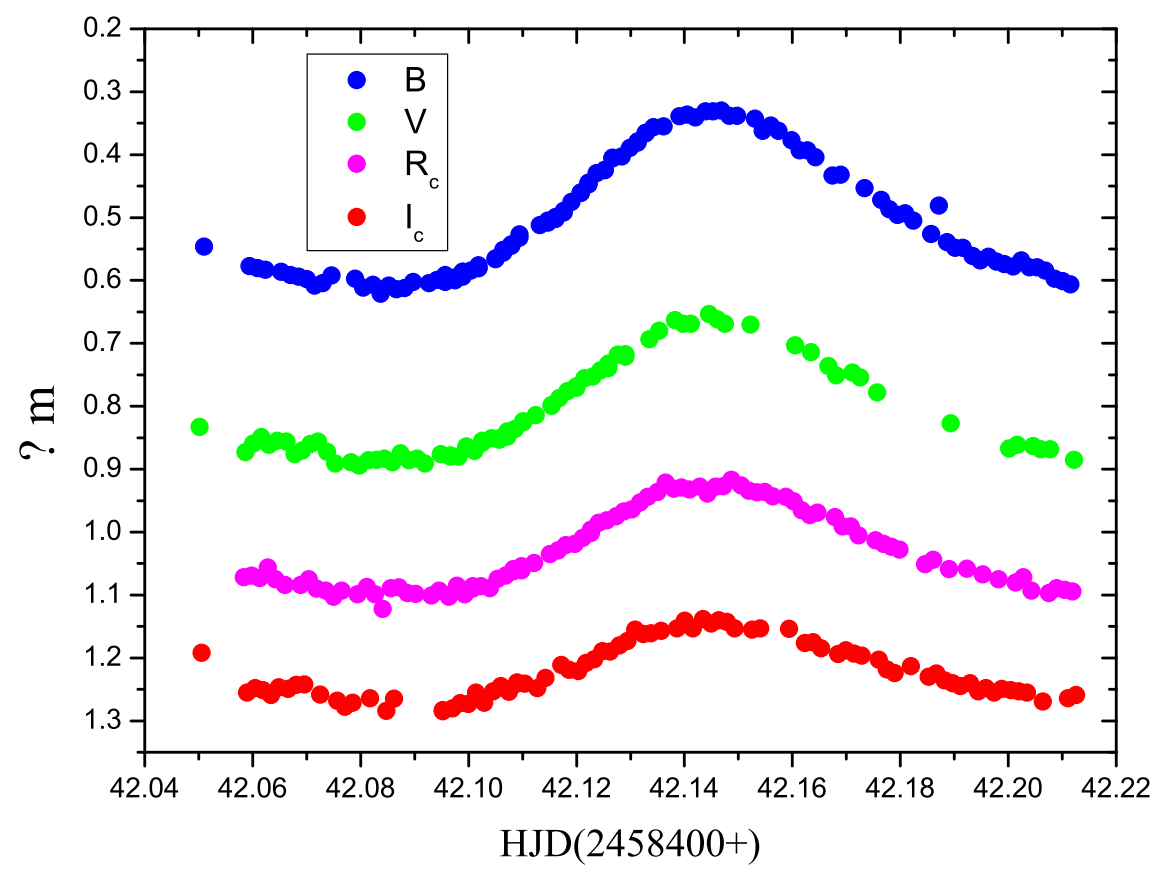

Figure 7: Light curves in $B V R_{c} I_{c}$ bands observed on November 19, 2018 with the 2.4-m telescope.

for the project has been provided by the National Development and Reform Commission. LAMOST is operated and managed by the National Astronomical Observatories, Chinese Academy of Sciences. Spectroscopic observations of those variables and binary stars used in the paper were obtained with LAMOST from October 24, 2011 to July 16, 2017. New CCD photometric observations were obtained with the $2.4 \mathrm{~m}$ telescope at the Yunnan Observatories and the $85-\mathrm{cm}$ telescope in Xinglong Observational station in China.

\section{References}

[1] Andersen, J. 1991, A\&ARv, 3, 91

[2] Torres, G., Andersen, J., \& Giménez, A. 2010, A\&ARv, 18, 67

[3] Drake, A. J., Djorgovski, S. G., Mahabal, A., Beshore, E., Larson, S., Graham, M. J., Williams, R., et al., 2009, ApJ 696, 870

[4] Drake, A. J., Graham, M. J., Djorgovski, S. G., Catelan, M., Mahabal, A. A., Torrealba, G., et al., 2014, ApJS 213, 9

[5] Palaversa, L., Ivezic, Z., Eyer, L., Ruzdjak, D., Sudar, D., Galin, M., Kroflin, A., et al., 2013, AJ 146, 101

[6] Pojmanski, G., 1997, AcA 47467

[7] Pojmanski, G., Pilecki, B., and Szczygiel, D., 2005, AcA 55, 275 
[8] Wozniak, P. R., Vestrand, W. T., Akerlof, C. W., Balsano, R., Bloch, J., Casperson, D., Fletcher, S., et al., 2004, AJ 127, 2436

[9] Watson, C. L., 2006, Society for Astronomical Sciences Annual Symposium, 25, 47

[10] Wang, S. G., Su, D. Q., Chu, Y. Q., et al. 1996, Appl. Opt., 35, 5155

[11] Cui, X. Q., Zhao, Y. H., Chu, Y. Q., et al. 2012, RAA 12, 1197

[12] Zhao, G., Zhao, Y. H., Chu, Y. Q., et al. 2012, RAA 12, 723

[13] Luo, A.-L., Zhao, Y. H., Zhao, G., et al. 2015, RAA 15, 1095

[14] Qian, S.-B., Li, L.-J., He, J.-J., Zhang, J., Zhu, L.-Y., Han, Z.-T., 2018a, MNRAS 475, 478

[15] Qian, S.-B., Li, L.-J., He, J.-J., Zhang, J., Zhu, L.-Y., Han, Z.-T., 2019, RAA 19, 1

[16] Qian S.-B., He J.-J., Zhang J., Zhu L.-Y., Shi X.-D., Zhao E.-G., and Zhou X., 2017, RAA 17, 87

[17] Qian, S.-B., Zhang, J., He, J.-J., Zhu, L.-Y., Zhao, E.-G., Shi, X.-D., Zhou, X., Han, Z.-T, 2018b, ApJS 235, 5

[18] Koleva, M., Prugniel, P., Bouchard, A. and Wu, Y. 2009, A\&A, 501, 1269

[19] Gao, H., Zhang, H.-W., Xiang, M.-S., et al., 2015, RAA 15, 2204

[20] Straizys, V., \& Kuriliene, G., 1981, Ap\&SS 80, 353

[21] Cox, A. N., 2000, Allen's Astrophysical Quantities, 4th ed. (New York: Springer), P.381

[22] Akerlof, C., Amrose, S., Balsano, R., Bloch, J., Casperson, D., Fletcher, S., et al., 2000, AJ 119, 1901

[23] Rodríguez, E., \& Breger, M., 2001, A\&A 366, 178

[24] Xiong, D. R., Deng, L., Zhang, C., \& Wang, K., 2016, MNRAS 457, 3163

[25] Zhou, A.-Y., Jiang, X.-J., Zhang, Y.-P., \& Wei, J.-Y. 2009, RAA 9, 349

[26] O’Connell, D. J. K., 1951, Publications of the Riverview College Observatory, 2, 85

[27] Brown, W. R., Beers, T. C., Wilhelm, R., Allende P., C., et al., 2008, AJ 135, 564 